\title{
Metodología para el seguimiento y evaluación global de las prácticas de laboratorio: experiencia en "Técnicas Analíticas del Medio Ambiente"
}

\section{M.J. Muñoz-Portero}

Departamento de Ingeniería Química y Nuclear, Universitat Politècnica de València, Camino de Vera s/n, 46022, Valencia (SPAIN), mjmunoz@iqn.upv.es, Equipo de Innovación y Calidad Educativa sobre Técnicas de Evaluación Continua Formativa (TECOF)

\begin{abstract}
The aim of the present work is the proposal of a methodology for global tracking and evaluation of the laboratory practices in a subject in the area of knowledge of Chemical Engineering, specifically in "Analytical Techniques of the Environment". The evaluation of the laboratory practices is proposed in three stages: 1) before of the practice session, 2) during the practice session, and 3) after of the practice session. In the present work different evaluation tools of the laboratory practices are shown. The pretests before the session of the laboratory practices allow to assess if the students have the necessary minimum knowledges for the realization of the laboratory practices. The rubrics filled during the session of the laboratory practices allow to the lecturer to assess the process that the students follow during the work in the laboratory. Finally, the postests after the session of the laboratory practices allow to assess if the students have achieved the required knowledges after the session of the laboratory practices. A global evaluation of the laboratory practices is expected to be achieved, evaluating both the final product and all the process of the realization of the practices.
\end{abstract}

Keywords: Evaluation, observation, e-learning platform, laboratory practice, rubric, test. 
Metodología para el seguimiento y evaluación global de las prácticas de laboratorio: experiencia en "Técnicas Analíticas del Medio Ambiente"

\section{Resumen}

El objetivo del presente trabajo es la propuesta de una metodología para el seguimiento y evaluación global de las prácticas de laboratorio en una asignatura del área de Ingeniería Química, concretamente en "Técnicas Analíticas del Medio Ambiente”. Para ello se propone realizar la evaluación de las prácticas de laboratorio en tres fases: 1) antes de la sesión de prácticas, 2) durante la sesión de prácticas y 3) después de la sesión de prácticas. En el presente trabajo se muestran distintos instrumentos de evaluación de las prácticas de laboratorio. Los pretests antes de la sesión de prácticas de laboratorio permiten evaluar si los alumnos disponen de los conocimientos mínimos necesarios para la realización de las prácticas de laboratorio. Las rúbricas rellenadas durante la sesión de prácticas de laboratorio permiten al profesorado evaluar el proceso que siguen los alumnos durante el trabajo en el laboratorio. Finalmente, los postests después de la sesión de prácticas de laboratorio permiten evaluar si los alumnos han adquirido los conocimientos requeridos tras la sesión de prácticas de laboratorio. Con ello se pretende conseguir una evaluación global de las prácticas de laboratorio, evaluando no solo el producto final sino también todo el proceso de realización de las prácticas.

Palabras clave: Evaluación, observación, plataforma de e-learning, práctica de laboratorio, rúbrica, test. 


\section{Introducción}

El nuevo escenario de la Educación Superior en el que nos encontramos está basado en un nuevo modelo educativo orientado al desarrollo de competencias y no únicamente a la adquisición de conocimientos, lo cual hace necesario la revisión del sistema de evaluación tradicional. Una buena evaluación es clave puesto que condiciona la calidad del aprendizaje. Hasta ahora las prácticas evaluativas tradicionales, basadas fundamentalmente en una prueba final, proporcionaban poca o ninguna retroalimentación tanto al profesor como al alumno. Por ello se hace necesario la búsqueda de nuevas estrategias de evaluación que favorezcan la motivación del alumno y la retroalimentación entre alumno y profesor (Díaz, 2009).

Una característica importante de la docencia en las carreras técnicas es que una parte importante de la misma son las clases prácticas. En ellas los alumnos ven la aplicación de conceptos teóricos y, además, adquieren una serie de habilidades actitudinales y procedimentales. Una problemática detectada en las sesiones de prácticas es que muchos alumnos asisten a las mismas sin un mínimo trabajo previo de lectura y revisión de los guiones de las prácticas. Por ello, se hace necesaria la búsqueda de herramientas para evaluar si los alumnos disponen de los conocimientos mínimos requeridos para la realización de las prácticas. En este sentido, la realización de cuestionarios mediante las plataformas docentes utilizadas en muchas universidades (PoliformaT en el caso de la UPV) puede ser de gran utilidad (Lowry, 2005; Martí, 2012; Nicholls, 1999). Dicho problema se está abordando desde el curso 2010-2011 gracias al Proyecto de Innovación y Mejora Educativa (PIME) sobre "Uso de la plataforma de e-learning PoliformaT para la evaluación de los conocimientos previos del alumno en las prácticas” (Ref. A011/10), concedido por la Universitat Politècnica de València (UPV).

Una segunda problemática detectada es que la evaluación de las actividades prácticas suele realizarse habitualmente mediante una prueba o trabajo tras la sesión de prácticas. Sin embargo, la evaluación del producto resultante de la sesión no es una evaluación global, ya que los alumnos no ponen interés en aprender los procedimientos seguidos, lo cual es, en la gran mayoría de los casos, lo más importante, ya que los procedimientos pueden extrapolarse a otras situaciones que puedan encontrarse en la vida profesional. Por tanto, se hace necesaria la observación del comportamiento del alumno durante la sesión de prácticas, es decir, evaluar el proceso de la sesión. Las rúbricas pueden ser una herramienta muy interesante para conseguirlo (Blanco, 2008; Boud, 2006; Johnson, 2007). Dicha problemática se está abordando desde el curso 2011-2012 gracias al PIME sobre "Uso de las rúbricas para el seguimiento y evaluación de las prácticas” (Ref. A16/11), concedido por la UPV.

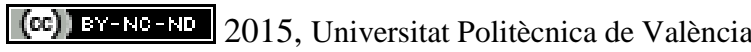

Congreso In-Red (2015) 
Metodología para el seguimiento y evaluación global de las prácticas de laboratorio: experiencia en "Técnicas Analíticas del Medio Ambiente"

Una tercera problemática detectada en las prácticas es cómo evaluar los conocimientos adquiridos por los alumnos tras la sesión de prácticas, de forma que los alumnos conozcan la calificación lo antes posible. De esta forma se pueden establecer mejoras de cara a próximas sesiones de prácticas favoreciendo la retroalimentación entre alumno y profesor. Esta problemática se está abordando desde el curso 2012-2013 gracias al PIME sobre "Evaluación de los conocimientos adquiridos por los alumnos en las prácticas a través del uso de la plataforma de e-learning PoliformaT” (Ref. A14/12), concedido por la UPV.

La metodología propuesta en el presente trabajo para el seguimiento y evaluación global de las prácticas de laboratorio se ha desarrollado de forma gradual gracias a los 3 PIMEs comentados anteriormente. Dichos PIMEs presentan un carácter multidisciplinar ya que participan un total de 10 asignaturas de diferentes titulaciones. Sin embargo, en el presente trabajo se muestran solo los resultados obtenidos en una asignatura del área de Ingeniería Química.

\section{Objetivos}

El objetivo del presente trabajo es la propuesta de una metodología para el seguimiento y evaluación global de las prácticas de laboratorio en una asignatura del área de Ingeniería Química. Para ello se propone realizar la evaluación de las prácticas de laboratorio en tres fases: 1) antes de la sesión de prácticas, 2) durante la sesión de prácticas y 3) después de la sesión de prácticas. De esa forma que se pretende conseguir una evaluación global de las prácticas de laboratorio.

\section{Desarrollo de la innovación}

\subsection{Contexto}

La metodología propuesta para el seguimiento y evaluación global de las prácticas de laboratorio se ha aplicado en la asignatura de "Técnicas Analíticas del Medio Ambiente (TAM)" de $5^{\circ}$ curso de la titulación de Ingeniero Químico en la Escuela Técnica Superior de Ingenieros Industriales (ETSII) de la UPV. Se trata de una asignatura optativa en la intensificación de Medioambiente de carácter semestral y consta de 5 créditos: 2,5 créditos de teoría, 1,4 créditos de prácticas de aula y 1,1 créditos de prácticas de laboratorio. El número de alumnos en los últimos cursos académicos oscila entre 3 y 10 alumnos. Las prácticas de laboratorio representan un $20 \%$ de la nota final de la asignatura. La metodología propuesta se ha aplicado en dos de las prácticas de laboratorio impartidas. 


\subsection{Metodología}

La metodología tradicional para la evaluación de las prácticas de laboratorio consistía únicamente en la realización de una memoria-informe después de la sesión de prácticas. Sin embargo, la evaluación del producto resultante de la sesión no era una evaluación global. Por ello, se hacía necesario buscar otras estrategias que permitieran evaluar todo el proceso de realización de las prácticas de laboratorio.

La metodología propuesta en el presente trabajo consiste en la evaluación de las prácticas de laboratorio en tres fases:

1) Antes de la sesión de prácticas. Para ello los alumnos realizan pretests utilizando la plataforma de e-learning PoliformaT, los cuales representan un $10 \%$ de la nota de la práctica.

2) Durante la sesión de prácticas. Para ello el profesor realiza la observación del trabajo de los alumnos en el laboratorio evaluando mediante rúbricas (10 \% de la nota de la práctica).

3) Después de la sesión de prácticas. Para ello los alumnos realizan postests utilizando la plataforma de e-learning PoliformaT, los cuales representan un $10 \%$ de la nota de la práctica. La nota de la práctica se completa con la entrega de una memoria-informe tras la realización de la sesión de prácticas (70 \% de la nota de la práctica).

\section{Resultados}

\subsection{Instrumentos de evaluación}

En el presente trabajo se muestran los diferentes instrumentos de evaluación diseñados para el seguimiento y evaluación global de las prácticas de laboratorio de la asignatura de "Técnicas Analíticas del Medio Ambiente" en las tres fases propuestas en los cursos académicos 2012-2013 y 2013-2014.

\subsubsection{Pretests}

Respecto a la primera fase, antes de la sesión de prácticas, los pretests se diseñan utilizando la herramienta "Exámenes" de la plataforma de e-learning PoliformaT. El pretest consiste en 5 preguntas de respuesta múltiple con 4 opciones de respuesta, de las cuales solo una es válida. Las 5 preguntas del pretest son seleccionadas aleatoriamente por el programa a partir de una batería de 10 preguntas. El tiempo máximo de duración del pretest es de 5 minutos. Los alumnos disponen de un plazo de 3 días justo antes de la práctica para rellenar el pretest. Cada alumno puede repetir el pretest hasta un máximo de 3 veces. De todos los intentos realizados se guardará la nota más alta obtenida por el alumno, la cual conocen los alumnos inmediatamente a través de la herramienta "Calificaciones" de la 
Metodología para el seguimiento y evaluación global de las prácticas de laboratorio: experiencia en "Técnicas Analíticas del Medio Ambiente"

plataforma de e-learning PoliformaT. La calificación obtenida será: “apto”, cuando se tiene un número de aciertos igual o superior a 4, y "no apto", cuando se tiene un número de aciertos igual o inferior a 3 o bien cuando no se realiza el test. Los alumnos con una calificación de “apto" en el pretest obtienen 1 punto sobre 10 en la nota de la práctica de laboratorio (10\% de la nota).

Los pretests permiten evaluar si los alumnos disponen de los conocimientos mínimos necesarios para la realización de las prácticas de laboratorio. A modo de ejemplo, en la Fig.1 se muestra un pretest utilizando la plataforma de e-learning PoliformaT, concretamente para la práctica de laboratorio sobre "determinación de fluoruros por potenciometría”.

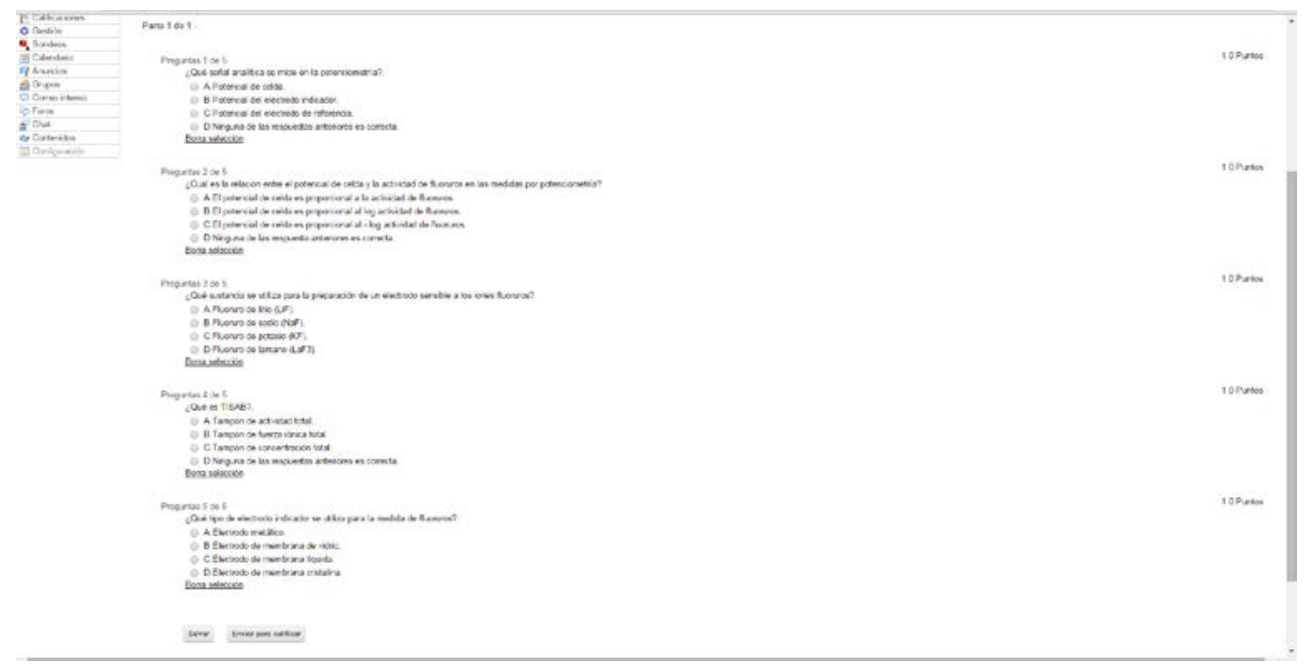

Fig. 1. Ejemplo de pretest utilizando la plataforma de e-learning PoliformaT para la evaluación de los conocimientos previos a las prácticas de laboratorio en "Técnicas Analíticas del Medio Ambiente".

\subsubsection{Rúbricas}

Respecto a la segunda fase, durante la sesión de prácticas, las rúbricas se diseñan estableciendo los indicadores de calidad a evaluar, que se agrupan en 3 bloques diferenciados: I) actitud, II) procedimientos (generales y específicos) y III) normas de seguridad y gestión de residuos. El bloque I de actitud presenta 3 indicadores de calidad: 1) participación e interés, 2) atención y 3) puntualidad. El bloque II de procedimientos se divide en 2 bloques: II.1) procedimientos generales y II.2) procedimientos específicos. El bloque II.1. de procedimientos generales presenta 3 indicadores de calidad, los cuales son comunes a todas las prácticas: 1) ritmo de trabajo, 2) destreza y autonomía y 3) recopilación de datos, cálculos e interpretación de resultados in situ. El bloque II.2 de procedimientos específicos tiene 3 o 4 indicadores de calidad diferentes en función de la 
práctica. Por último, el bloque III de normas de seguridad y gestión de residuos contiene sólo un indicador de calidad a valorar. Los criterios de evaluación en las rúbricas se establecen en una escala de 0 a 3: mal (0), regular (1), bien (2) y excelente (3).

Las rúbricas están disponibles en la herramienta "Recursos" de la plataforma de e-learning PoliformaT previamente a cada sesión de prácticas. De este modo, el alumno puede conocer cuáles son los indicadores que van a considerarse en su evaluación. Las rúbricas son cumplimentadas por el profesor durante la sesión de prácticas para verificar si se cumplen cada uno de los indicadores de calidad propuestos. Aunque los alumnos se distribuyen por equipos para realizar la práctica, en el caso de la asignatura de "Técnicas Analíticas del Medio Ambiente” las rúbricas se utilizan para evaluar a los alumnos de forma individual. El profesor establece retroalimentación con el alumno al inicio de la siguiente sesión de prácticas. El profesor pone en conocimiento de los alumnos de forma individual los aspectos a mejorar que se derivan de sus rúbricas de la práctica anterior para que los tengan en cuenta en esa sesión.

Las rúbricas permiten al profesor evaluar el proceso que siguen los alumnos en la sesión de prácticas de laboratorio para realizar el trabajo que se les propone. En el Anexo 1 se muestra un ejemplo de rúbrica para el seguimiento y evaluación de las prácticas de laboratorio, concretamente para la práctica de laboratorio sobre "determinación de $\mathrm{NO}_{\mathrm{x}} \mathrm{y}$ partículas en humo de tabaco".

\subsubsection{Postests}

Respecto a la tercera fase, después de la sesión de prácticas, los postests se diseñan siguiendo el mismo formato que los pretests. En este caso los alumnos disponen de un plazo de 3 días justo después de la práctica para rellenar el postest. Los alumnos con una calificación de "apto" en el postest obtienen 1 punto sobre 10 en la nota de la práctica de laboratorio (10\% de la nota).

Los postests permiten evaluar si los alumnos han adquirido los conocimientos requeridos después de la sesión de prácticas de laboratorio. En la Fig. 2 se muestra un ejemplo de postest utilizando la plataforma de e-learning PoliformaT, concretamente para la práctica de laboratorio sobre "determinación de $\mathrm{NO}_{\mathrm{x}}$ y partículas en humo de tabaco".

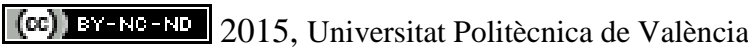

Congreso In-Red (2015) 
Metodología para el seguimiento y evaluación global de las prácticas de laboratorio: experiencia en "Técnicas Analíticas del Medio Ambiente"

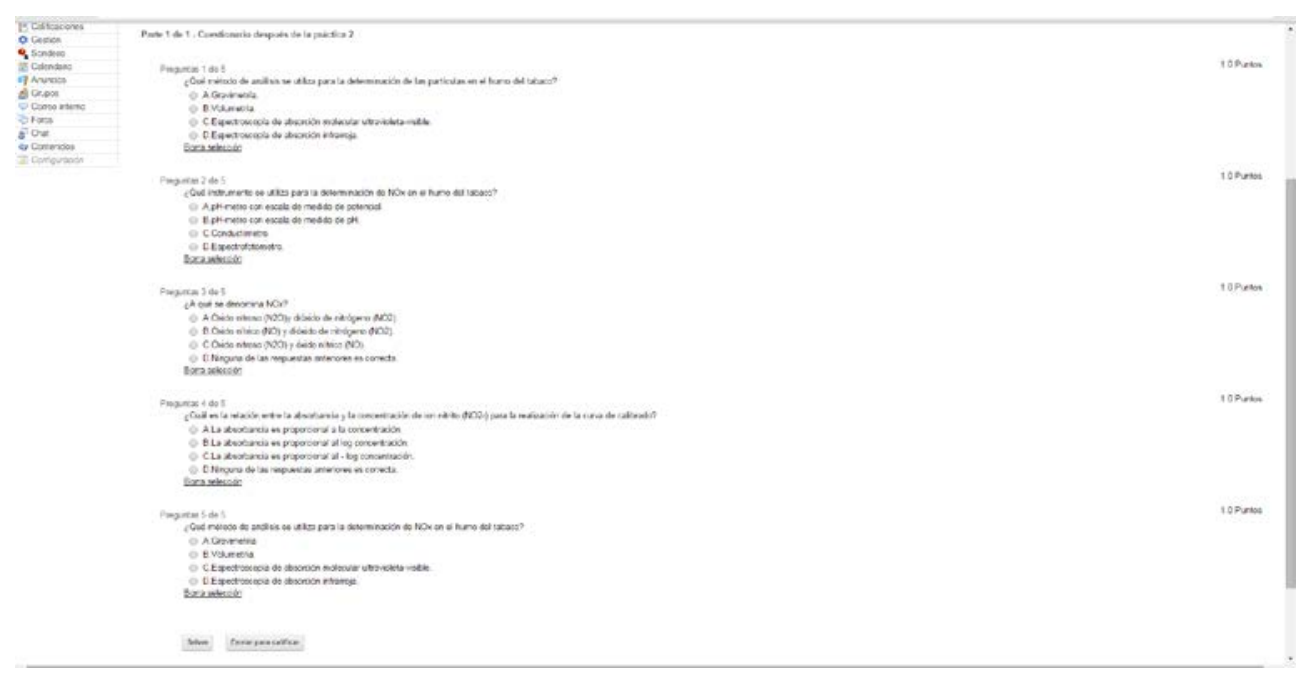

Fig. 2. Ejemplo de postest utilizando la plataforma de e-learning PoliformaT para la evaluación de los conocimientos adquiridos después de las prácticas de laboratorio en "Técnicas Analíticas del Medio Ambiente".

\subsection{Calificaciones de los alumnos}

En la Tabla 1 se muestran las calificaciones obtenidas por los alumnos en las prácticas de laboratorio en la asignatura de "Técnicas Analíticas del Medio Ambiente" desde el curso 2010-2011 hasta el curso 2013-2014. En dicha tabla se incluye para cada curso académico el sistema de evaluación aplicado en las prácticas de laboratorio, la nota de cada una de las prácticas de laboratorio y la nota media.

Los resultados mostrados en la Tabla 1 indican que las calificaciones de los alumnos han ido aumentando a medida que se han ido introduciendo las distintas fases de la nueva metodología propuesta. Así, en el curso 2010-2011, en el cual solo se habían implantado los pretests (primera fase), se obtiene la calificación más baja, con un valor medio de 8,11, mientras que en el curso 2013-2014, en el cual ya se habían implantado todas las fases de la nueva metodología, se obtiene la calificación más alta, con un valor medio de 9,39.

Con la introducción de diferentes instrumentos de evaluación en las prácticas de laboratorio (pretests, rúbricas y postests) se pretende conseguir una evaluación global de las prácticas de laboratorio, evaluando no solo el producto final sino también todo el proceso de realización de las prácticas de laboratorio. 
Tabla 1. Calificaciones obtenidas por los alumnos en las prácticas de laboratorio en la asignatura de "Técnicas Analíticas del Medio Ambiente”.

\begin{tabular}{|c|c|c|c|c|}
\hline Curso académico & Sistema de evaluación & Práctica $1^{*}$ & Práctica $2^{* *}$ & Media \\
\hline 2010-2011 & $\begin{array}{c}\text { Memoria-informe (90 \%) } \\
\text { Pretest (10 \%) }\end{array}$ & 7,70 & 8,52 & 8,11 \\
\hline 2011-2012 & $\begin{array}{c}\text { Memoria-informe (80 \%) } \\
\text { Pretest (10 \%) } \\
\text { Observación-Rúbrica (10 \%) }\end{array}$ & 8,40 & 8,80 & 8,60 \\
\hline 2012-2013 & $\begin{array}{l}\text { Memoria-informe (70 \%) } \\
\text { Pretest (10 \%) } \\
\text { Observación-Rúbrica (10 \%) } \\
\text { Postest (10 \%) }\end{array}$ & 8,29 & 9,07 & 8,68 \\
\hline 2013-2014 & $\begin{array}{l}\text { Memoria-informe (70 \%) } \\
\text { Pretest (10 \%) } \\
\text { Observación-Rúbrica (10 \%) } \\
\text { Postest (10 \%) }\end{array}$ & 9,39 & 9,39 & 9,39 \\
\hline
\end{tabular}

* Práctica 1: Determinación de $\mathrm{NO}_{\mathrm{x}}$ y partículas en humo de tabaco.

*** Práctica 2: Determinación de fluoruros por potenciometría.

\section{Conclusiones}

En este trabajo se han mostrado distintos instrumentos de evaluación que permiten resolver la problemática que presenta la evaluación de las prácticas de laboratorio. Los pretests realizados antes de la sesión de prácticas de laboratorio permiten evaluar si los alumnos disponen de los conocimientos mínimos necesarios para la realización de las prácticas de laboratorio. Las rúbricas rellenadas durante la sesión de prácticas de laboratorio permiten al profesorado evaluar el proceso que siguen los alumnos durante el trabajo en el laboratorio. Finalmente, los postests realizados después de la sesión de prácticas de laboratorio permiten evaluar si los alumnos han adquirido los conocimientos requeridos tras la sesión de prácticas de laboratorio. Con ello se pretende conseguir una evaluación global de las prácticas de laboratorio, evaluando no solo el producto final sino también todo el proceso de realización de las prácticas de laboratorio. 
Metodología para el seguimiento y evaluación global de las prácticas de laboratorio: experiencia en "Técnicas Analíticas del Medio Ambiente"

\section{Referencias}

BLANCO, A. (2008). "Las rúbricas un instrumento útil en la evaluación de competencias" en Prieto, L. (Coord.), Blanco, A., Morales, P., Torre, J.C. La enseñanza universitaria centrada en el aprendizaje: estrategias útiles para el profesorado. Barcelona: OctaedroICE de la Universidad de Barcelona.

BOUD, D. (2006). “Foreword” en Bryan, C., Clegg, K. (Ed.) Innovative Assessment in Higher Education. Londres: Routledge, p. XVII-XIX.

DIAZ, T. (2009). "Experiencia de innovación docente sobre estrategias de evaluación que mejoran la motivación del alumno en el proceso de aprendizaje”. I Congreso de Docencia Universitaria. Universidad de Vigo.

JOHNSON, A., SVINGBY, G. (2007). "The use of rubrics: reliability, validity, and educational consequences” en Educational Research Review, 2, p. 130-144.

LOWRY, R (2005). “Computer aided self assessment-an effective tool” en Chemistry Education Research and Practice, 6, 4, p.198-203.

MARTÍ, N. et al. (2012). "Evaluación y seguimiento del estudiante en el grado de Ingeniería Química: Uso de cuestionarios en plataforma e-learning". I Congreso de Innovación Docente en Ingeniería Química. Universidad de Granada.

NICHOLLS, B.S. (1999). "Prelaboratory support using dedicated software” en University Chemistry Education, 3, p. 22-27. 
Anexo 1. Ejemplo de rúbrica para el seguimiento y evaluación de las prácticas de laboratorio en la asignatura de "Técnicas Analíticas del Medio Ambiente".

\begin{tabular}{|c|c|c|c|c|}
\hline & Mal (0) & Regular (1) & Bien (2) & Excelente (3) \\
\hline \multicolumn{5}{|l|}{ I. ACTITUD } \\
\hline Participación e interés & $\begin{array}{l}\text { Tiene una actitud } \\
\text { pasiva o negativa. No } \\
\text { muestra interés en el } \\
\text { transcurso de la } \\
\text { práctica. No responde a } \\
\text { las preguntas del } \\
\text { profesor. }\end{array}$ & 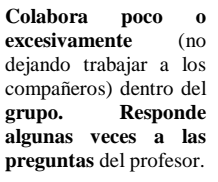 & $\begin{array}{l}\text { Colabora dentro del } \\
\text { grupo. Responde a las } \\
\text { preguntas del profesor } \\
\text { y algunas veces realiza } \\
\text { preguntas. }\end{array}$ & $\begin{array}{l}\text { Tiene una actitud } \\
\text { activa y positiva } \\
\text { dentro del grupo y en } \\
\text { la clase. Responde a } \\
\text { las preguntas del } \\
\text { profesor y realiza } \\
\text { también preguntas. }\end{array}$ \\
\hline Atención & $\begin{array}{l}\text { No presta atención: } \\
\text { está distraído haciendo } \\
\text { otras cosas, no responde } \\
\text { a las llamadas de } \\
\text { atención del profesor o } \\
\text { molesta a los } \\
\text { compañeros. }\end{array}$ & $\begin{array}{lr}\text { Se distrae a veces } \\
\text { durante } & \text { las } \\
\text { explicaciones } & \text { del } \\
\text { profesor y durante la } \\
\text { realización de las } \\
\text { tareas. }\end{array}$ & $\begin{array}{lr}\text { Se muestra siempre } \\
\text { atento durante las } \\
\text { explicaciones del } \\
\text { profesor y durante la } \\
\text { realización de las } \\
\text { tareas, pero sin tomar } \\
\text { notas de las } \\
\text { explicaciones. }\end{array}$ & $\begin{array}{l}\text { Se muestra siempre } \\
\text { atento durante las } \\
\text { explicaciones des } \\
\text { profesor y durante la } \\
\text { realización de las } \\
\text { tareas, tomando notas } \\
\text { de las explicaciones. }\end{array}$ \\
\hline Puntualidad & $\begin{array}{l}\text { Asiste con un retraso } \\
\text { superior a } 10 \text { minutos. }\end{array}$ & $\begin{array}{l}\text { Asiste con un retraso } \\
\text { inferior a } 10 \text { minutos. }\end{array}$ & $\begin{array}{l}\text { Asiste con } \\
\text { puntualidad, pero no } \\
\text { está preparado para } \\
\text { empezar a trabajar } \\
\text { cuando lo indica el } \\
\text { profesor. }\end{array}$ & $\begin{array}{lr}\text { Asiste } & \text { con } \\
\text { puntualidad y está } \\
\text { preparado para } \\
\text { empezar a trabajar } \\
\text { cuando lo indica el } \\
\text { profesor. }\end{array}$ \\
\hline \multicolumn{5}{|l|}{ II. PROCEDIMIENTOS } \\
\hline \multicolumn{5}{|l|}{ II.1. Procedimientos generals } \\
\hline Ritmo de trabajo & $\begin{array}{l}\text { No trabaja durante la } \\
\text { práctica. }\end{array}$ & $\begin{array}{l}\text { Trabaja de forma } \\
\text { discontinua durante la } \\
\text { práctica realizando } \\
\text { parones. }\end{array}$ & $\begin{array}{l}\text { Trabaja de forma } \\
\text { constante durante la } \\
\text { práctica, pero sin } \\
\text { aprovechar los } \\
\text { tiempos "muertos". }\end{array}$ & $\begin{array}{l}\text { Trabaja de forma } \\
\text { constante durante la } \\
\text { práctica aprovechando } \\
\text { los } \\
\text { "muertos". }\end{array}$ \\
\hline Destreza y autonomía & $\begin{array}{l}\text { No tiene un buen } \\
\text { manejo del material y } \\
\text { los equipos de } \\
\text { laboratorio. Se dedica a } \\
\text { "copiar" } \\
\text { continuamente lo que } \\
\text { hacen los otros } \\
\text { compañeros. }\end{array}$ & $\begin{array}{l}\text { Tiene un manejo } \\
\text { básico del material y } \\
\text { los equipos de } \\
\text { laboratorio, limitándose } \\
\text { en gran medida a seguir } \\
\text { el guión de la práctica. } \\
\text { Ante cualquier } \\
\text { problema recurre al } \\
\text { profesor o "copia” lo } \\
\text { que hacen otros } \\
\text { compañeros (no } \\
\text { intenta solucionarlo por } \\
\text { sí mismo). }\end{array}$ & $\begin{array}{l}\text { Tiene un buen manejo } \\
\text { del material y los } \\
\text { equipos de laboratorio, } \\
\text { y en la medida de lo } \\
\text { posible intenta } \\
\text { resolver por sí mismo } \\
\text { los problemas que van } \\
\text { apareciendo. }\end{array}$ & $\begin{array}{l}\text { Tiene un manejo } \\
\text { avanzado del material } \\
\text { y los equipos de } \\
\text { laboratorio, y es capaz } \\
\text { de resolver por sí } \\
\text { mismo los problemas } \\
\text { que van apareciendo. }\end{array}$ \\
\hline $\begin{array}{l}\text { Recopilación de datos, cálculos } \\
\text { e interpretación de resultados } \\
\text { in situ }\end{array}$ & $\begin{array}{l}\text { Recopila los datos en el } \\
\text { laboratorio de } \text { forma } \\
\text { desordenada. } \\
\text { realiza los cálculos } \\
\text { previos ni r la } \\
\text { interpretación de los } \\
\text { resultados in situ. }\end{array}$ & $\begin{array}{l}\text { Recopila los datos en } \\
\text { el laboratorio de forma } \\
\text { parcialmente } \\
\text { ordenada. Realiza los } \\
\text { cálculos previos y la } \\
\text { interpretación de los } \\
\text { resultados in situ de } \\
\text { forma incorrecta o } \\
\text { bien de forma correcta } \\
\text { con bastante ayuda } \\
\text { del profesor o } \\
\text { copiándose de otros } \\
\text { compañeros. }\end{array}$ & $\begin{array}{l}\text { Recopila los datos en } \\
\text { el laboratorio de forma } \\
\text { ordenada. Realiza los } \\
\text { cálculos previos y la } \\
\text { interpretación de los } \\
\text { resultados in situ de } \\
\text { forma correcta, pero } \\
\text { con algo de ayuda del } \\
\text { profesor. }\end{array}$ & $\begin{array}{l}\text { Recopila los datos en } \\
\text { el laboratorio de forma } \\
\text { muy ordenada. } \\
\text { Realiza los cálculos } \\
\text { previos y la } \\
\text { interpretación de los } \\
\text { resultados in situ de } \\
\text { forma correcta sin la } \\
\text { ayuda del profesor. }\end{array}$ \\
\hline
\end{tabular}

(cc)) EY-NC-ND 2015, Universitat Politècnica de València

Congreso In-Red (2015) 
Metodología para el seguimiento y evaluación global de las prácticas de laboratorio: experiencia en "Técnicas Analíticas del Medio Ambiente"

\begin{tabular}{|c|c|c|c|c|}
\hline \multicolumn{5}{|l|}{ II.2. Procedimientos específicos } \\
\hline $\begin{array}{l}\text { Muestreo de partículas en el } \\
\text { humo del tabaco }\end{array}$ & $\begin{array}{l}\text { Realiza de forma } \\
\text { incorrecta el muestreo } \\
\text { de partículas en el humo } \\
\text { del tabaco. }\end{array}$ & $\begin{array}{l}\text { Realiza de forma } \\
\text { correcta el muestreo } \\
\text { de partículas en el } \\
\text { humo del tabaco, } \\
\text { necesitando bastante } \\
\text { asesoramiento por } \\
\text { parte del profesor. }\end{array}$ & $\begin{array}{l}\text { Realiza de forma } \\
\text { correcta el muestreo de } \\
\text { partículas en el humo } \\
\text { del tabaco, necesitando } \\
\text { algo de asesoramiento } \\
\text { por parte del } \\
\text { profesor. }\end{array}$ & $\begin{array}{l}\text { Realiza de forma } \\
\text { correcta el muestreo de } \\
\text { partículas en el humo } \\
\text { del tabaco sin } \\
\text { asesoramiento por } \\
\text { parte del profesor. }\end{array}$ \\
\hline $\begin{array}{c}\text { Preparación de los patrones de } \\
\text { calibrado }\end{array}$ & $\begin{array}{l}\begin{array}{l}\text { Realiza de forma } \\
\text { incorrecta }\end{array} \\
\text { preparación de la } \\
\text { patrones de calibrado. }\end{array}$ & $\begin{array}{l}\text { Realiza de forma } \\
\text { correcta la } \\
\text { preparación de los } \\
\text { patrones de calibrado, } \\
\text { necesitando bastante } \\
\text { asesoramiento por } \\
\text { parte del profesor. }\end{array}$ & $\begin{array}{l}\text { Realiza de forma } \\
\text { correcta la preparación } \\
\text { de los patrones de } \\
\text { calibrado, necesitando } \\
\text { algo de asesoramiento } \\
\text { por parte del } \\
\text { profesor. }\end{array}$ & $\begin{array}{l}\text { Realiza de forma } \\
\text { correcta la preparación } \\
\text { de los patrones de } \\
\text { calibrado sin } \\
\text { asesoramiento por } \\
\text { parte del profesor. }\end{array}$ \\
\hline $\begin{array}{c}\text { Muestreo de } \mathrm{NO}_{\mathrm{x}} \text { en el humo } \\
\text { del tabaco }\end{array}$ & $\begin{array}{l}\text { Realiza de forma } \\
\text { incorrecta el muestreo } \\
\text { de } \mathrm{NO}_{\mathrm{x}} \text { en el humo del } \\
\text { tabaco. }\end{array}$ & $\begin{array}{l}\text { Realiza de forma } \\
\text { correcta el muestreo } \\
\text { de } \mathrm{NO}_{\mathrm{x}} \text { en el humo del } \\
\text { tabaco, necesitando } \\
\text { bastante } \\
\text { asesoramiento por } \\
\text { parte del profesor. }\end{array}$ & $\begin{array}{l}\text { Realiza de forma } \\
\text { correcta el muestreo de } \\
\mathrm{NO}_{\mathrm{x}} \text { en el humo del } \\
\text { tabaco, necesitando } \\
\text { algo de asesoramiento } \\
\text { por parte del } \\
\text { profesor. }\end{array}$ & $\begin{array}{l}\text { Realiza de forma } \\
\text { correcta el muestreo de } \\
\mathrm{NO}_{\mathrm{x}} \text { en el humo del } \\
\text { tabaco sin } \\
\text { asesoramiento por } \\
\text { parte del profesor. }\end{array}$ \\
\hline $\begin{array}{l}\text { Medida de la absorbancia con } \\
\text { el espectrofotómetro }\end{array}$ & $\begin{array}{l}\text { Realiza de forma } \\
\text { incorrecta la medida de } \\
\text { la absorbancia con el } \\
\text { espectrofotómetro. }\end{array}$ & $\begin{array}{l}\text { Realiza de forma } \\
\text { correcta la medida de } \\
\text { la absorbancia con el } \\
\text { espectrofotómetro con } \\
\text { bastante } \\
\text { asesoramiento por } \\
\text { parte del profesor. }\end{array}$ & $\begin{array}{l}\text { Realiza de forma } \\
\text { correcta la medida de } \\
\text { la absorbancia con el } \\
\text { espectrofotómetro con } \\
\text { algo de asesoramiento } \\
\text { por parte del } \\
\text { profesor. }\end{array}$ & $\begin{array}{l}\text { Realiza de forma } \\
\text { correcta la medida de } \\
\text { la absorbancia con el } \\
\text { espectrofotómetro sin } \\
\text { asesoramiento por } \\
\text { parte del profesor. }\end{array}$ \\
\hline \multicolumn{5}{|c|}{ III. NORMAS DE SEGURIDAD Y GESTIÓN DE RESIDUOS } \\
\hline $\begin{array}{l}\text { Seguridad en el laboratorio y } \\
\text { gestión de residuos }\end{array}$ & $\begin{array}{l}\text { Desconoce los datos de } \\
\text { seguridad de los } \\
\text { reactivos a utilizar en la } \\
\text { práctica. } \\
\text { No gestiona los } \\
\text { residuos. }\end{array}$ & $\begin{array}{l}\text { Conoce y aplica de } \\
\text { forma correcta los } \\
\text { datos de seguridad, } \\
\text { necesitando bastante } \\
\text { asesoramiento por } \\
\text { parte del profesor. } \\
\text { Gestiona los residuos } \\
\text { de forma incorrecta o } \\
\text { bien de forma correcta } \\
\text { con bastante ayuda } \\
\text { del profesor o } \\
\text { copiándose de otros } \\
\text { compañeros. }\end{array}$ & $\begin{array}{l}\text { Conoce y aplica de } \\
\text { forma correcta los } \\
\text { datos de seguridad, } \\
\text { necesitando algo de } \\
\text { asesoramiento por } \\
\text { parte del profesor. } \\
\text { Gestiona los residuos } \\
\text { de forma correcta con } \\
\text { algo de ayuda del } \\
\text { profesor }\end{array}$ & $\begin{array}{l}\text { Conoce y aplica de } \\
\text { forma correcta los } \\
\text { datos de seguridad sin } \\
\text { asesoramiento por } \\
\text { parte del profesor. } \\
\text { Gestiona los residuos } \\
\text { de forma correcta sin } \\
\text { la ayuda del profesor. }\end{array}$ \\
\hline
\end{tabular}

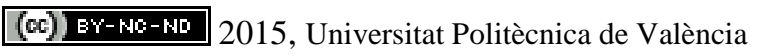

